\title{
Marija Pavelić: The invisible Female Leader of the Independent State of Croatia (1941-1945)
}

\author{
Martina Bitunjac, PhD \\ Sapienza-University of Rome \\ Martina.Bitunjac@uniroma1.it
}

\section{Doi:10.5901/mjss.2013.v4n2p205}

\section{Abstract:}

The Independent State of Croatia was established in 1941 with the help of Nazi Germany and fascist Italy after the Kingdom of Yugoslavia was destroyed and divided by the Axis powers. As an anti-Yugoslavian, anti-Communist and anti-Semitic organisation, the ustasha displayed very strong tendencies towards National Socialist and fascist ideology, incorporated it and implemented it as legislation. The dispossession and annihilation of Serbs, Jews, Roma and resistance fighters were the consequences of the totalitarian state that was headed by the ustasha cult figure Dr. Ante Pavelić. Also women played an important role in the creation of the new state, even if the ustasha in their propaganda practiced the centuries-old, conservative-Catholic patriarchy in a form that resembled Fascism in many aspects. This article aims to analyze the role of one of the most powerful women in the Independent State of Croatia: Marija Pavelić, the wife of the ustasha-leader. Although Pavelic's wife Marija gave birth to three children and rarely appeared in public, she was already a politically active woman in the Italian exile (1929-1941) and attempted to support the power of her husband from the beginning of his political occupation.

Keywords: Marija Pavelić, Independent State of Croatia, ustasha, Second World War.

\section{Introduzione: Le donne e il movimento ustascia}

II Movimento di Liberazione Croata degli Ustascia (Ustaša - Hrvatski oslobodilački pokret) fu fondato nel 1930 dall'avvocato Ante Pavelić durante il suo esilio in Italia come reazione al centralismo politico serbo nel Regno di lugoslavia. ${ }^{1}$ || ruolo delle donne nel "periodo di lotta" degli ustascia è stato di un'importanza enorme per la realizzazione degli obiettivi del movimento, in particolare per la fondazione di uno Stato indipendente croato, che fu proclamato nell'aprile 1941, sotto il dominio degli ustascia. Fino al 1941 Ante Pavelić e i suoi sostenitori, per motivi politico-strategici, non si impegnarono per l'isolamento delle donne dal movimento né si sforzarono per limitare le donne al loro ruolo tradizionale di madri. Le donne, che fino al 1941 avevano partecipato alla lotta per uno Stato indipendente croato, non avevano rotto soltanto l'ordine tradizionale dei ruoli tra uomini e donne ma nella loro funzione di mediatrici, corrieri, informatrici, spie e terroriste, erano dei membri del movimento ustascia, apprezzati nella cerchia d'élite intorno ad loro 'poglavnik' ('duce'). Tra le attiviste della prima ora vi era in particolare Marija Pavelić, la moglie del capo di Stato. Anche nello Stato totalitario degli ustascia (1941-1945) il ruolo delle donne in un mondo soprattutto maschile era di grande importanza sia nell'economia che nella politica e nell'ambiente militare. Marija Pavelić, oltre ad avere un ruolo rappresentativo nello stato totalitario, approfittava del suo potere e influenzò durante la guerra la politica ustascia, di cui alcune caratteristiche erano il sentimento razzista e lo sterminio dei serbi, degli ebrei e dei rom.

\section{Marija Pavelić durante l'esilio in Italia (1929-1941)}

L'interesse nei confronti della politica, Marija Pavelić, nata Lovrenčević, l'aveva già sviluppato nell'ambito della sua famiglia d'origine, essendo la figlia di Martin Lovrenčević, il celebre pubblicista e sostenitore di Ante Starčević, il

${ }^{1}$ Sullo Stato Indipendente di Croazia vedi: Tomasevich, J. (2010).Rat i revolucija u Jugoslaviji 1941.-1945. Okupacija i kolaboracija, Zagabria: EPH Media; Becherelli, A. (2012). Italia e Stato Indipendente Croato (1941-1943), Roma: Nuova cultura. 
fondatore del Partito del Diritto (Stranka prava). ${ }^{2}$ La madre di Marija Pavelić, Ivana Herzfeld, scomparsa nel 1942, era di origine ebrea e aveva lavorato come governante presso la famosa famiglia zagabrese dei Šram. Questa professione è stata, per molto tempo, una delle poche permesse alle giovani donne non sposate dei ceti medi per essere economicamente indipendenti. La Herzfeld si occupava dell'educazione dell'attrice Ljerka Šram e conobbe in questa casa il suo futuro marito, Martin Lovrenčević, che lavorava nello studio legale di Vjekoslav Šram e che era, al tempo stesso, il precettore dei suoi figli. Per il centro culturale Matica Hrvatska, Lovrenčević traduceva delle opere della letteratura mondiale dal russo, dall'inglese e dal francese al croato e, da sostenitore convinto di Starčević, collaborava alla redazione dei giornali del Partito del Diritto. Ivana Herzfeld e Martin Lovrenčević si sposarono ed ebbero cinque figlie: Anka, Zora, Vera, Nata e Marija.

Dopo essersi diplomata presso la scuola femminile cattolica delle Suore della Carità di San Vincenzo (Sestre milosrdnica Svetog Vinka)a Zagabria, Marija Lovrenčević frequentò un istituto commerciale, benché non potesse svolgere il mestiere della sarta in quanto suo padre desiderava che lei lavorasse come segretaria dell'avvocato e presidente del Partito del Diritto Aleksandar Horvat. Durante il tempo trascorso allo studio legale, la giovane conobbe il suo futuro marito, l'avvocato Ante Pavelić. Passarono molti anni, però, prima che la coppia si legasse in matrimonio, perché Pavelić, secondo le affermazioni di sua figlia maggiore Višnja Pavelić, era consapevole del fatto di riuscire a costruirsi una carriera politica soltanto con una donna forte ed equilibrata al suo fianco, che ragionava in termini politici come lui:

"Mio padre ci ha pensato a lungo, molto lungo. Per moltissimo tempo ci ha pensato, finché il vecchio [=Martin Lovrenčević] era costretto a dire: 'Che cosa è questo?' Al vecchio nonno tutto questo non piaceva. Ma si vede che ha esaminato minuziosamente mia madre, che ci si volevano molti anni, finché non ha deciso di sposarla. Poiché sapeva di entrare nella politica e non ha voluto avere scontri in famiglia. In questo senso, però, ha preso (la giusta decisione)."3

Ante Pavelić, già vice-segretario del Partito del Diritto e deputato dell'assemblea nazionale a Belgrado, diventò famoso nella sua funzione di avvocato difensore dei nazionalisti macedoni a Skopje, quando richiamò l'attenzione sul trattamento ingiusto dei popoli non-serbi e sull'abrogazione della libertà d'opinione in lugoslavia. Per la sua protesta pubblica, fu condannato a morte in absentia dal Tribunale di Stato per la Protezione dello Stato a Belgrado. Nel 1929, Pavelić fuggì nell'Italia fascista, dove avrebbe preparato la fondazione dello Stato degli ustascia sotto la protezione di Benito Mussolini. Marija Pavelić seguì suo marito nell'esilio italiano con i loro tre figli Višnja, Velimir e Mirjana. L'immagine di Marija Pavelić in esilio era - diversamente dal periodo in seguito alla presa di potere degli ustascia - quella di una moglie e madre distaccata dalla politica. In pubblico si faceva vedere raramente e in quelle rare occasioni si comportava in maniera riservata. Ante Moškov, il generale e comandante della Guardia del Corpo di Pavelić, durante la prigionia dopo la guerra dichiarò che Marija Pavelić era stata vista una volta sola dagli ustascia nell'esilio italiano. Era all'Aquila nel 1934, quando in compagnia di suo marito andò a vedere gli ustascia nel campo di formazione militare. Non avrebbe detto neanche una parola, ma avrebbe ascoltato i colloqui di suo marito. ${ }^{4}$ Solo pochi membri del movimento conoscevano la moglie del capo degli ustascia personalmente, la maggior parte di loro era formata da persone dell'élite del comando e dai loro familiari, che frequentavano i Pavelić nei loro domicili in Italia.

I figli di Marija Pavelić o crescevano in istituti educativi elitari o erano guardati dalla governante Maria Stefania Schicker, cosicché la Pavelić aveva abbastanza tempo per sostenere suo marito negli affari politici.S'impegnò intellettualmente per la costituzione dello Stato indipendente croato, redigendo il libro Per la Libertà della Croazia (Za slobodu Hrvatske), in collaborazione con suo marito e con il vice-segretario del Partito dei Contadini Croato (Hrvatska Seljačka Stranka) August Košutić. II libro tratta del processo contro i due avversari della dittatura iugoslava, Marko Hranilović e Matija Soldin, entrambi membri della Gioventù del Diritto Croata, proibita nel Regno di lugoslavia, condannati a morte per capestro per la loro avversione al regime nel 1931 (Jareb, 1982, p. 44). Oltre alle sue attività intellettuali,

2 Intervista con Višnja Pavelić a Madrid del 24. 2. 2008. Vedi anche: Pavelić, V. (2001). Martin Lovrenčević, Grič, 1, 25-32.

3 "Moj otac je dugo, dugo razmišljalo. Jako dugo je razmišljao, tako da je stari [=Martin Lovrenčević] morao govoriti: ‘Šta je ovo?'Starom djedu se nije sviđalo. Ali vidi se da je on proučavao moju mamu. I mislim da je to trajalo dosta godina dok se odlučio da je ženi. Jer on je znao da će on sa politikom imati posla i nije htio nešta doživiti u familiji.Ali u tom pogledu je pogodio." Intervista con Višnja Pavelića Madrid del 24. 2. 2008.

${ }^{4}$ Hrvatski Državni Arhiv (HDA), Služba državne sigurnosti republičkog sekretarijata za unutrašnje poslove Socijalističke Republike Hrvatske (SDS RSUP SRH) - 1561, Ustaški pokret, busta 24, sig. 013.0.4, pratica Ante Pavelić, deposizione di Ante Moškov, senza data. 
Marija Pavelić svolgeva altri compiti nell'interesse del movimento: quando Ante Pavelić, dall'ottobre 1934 al 1936, fu internato nel carcere di Torino per la partecipazione degli ustascia all'omicidio del re iugoslavo Aleksandar I. Karađorđević, sua moglie faceva uscire dal carcere degli ordini scritti del 'poglavnik', indirizzati ai suoi sostenitori, tenendoli nascosti dentro il cibo. Nelle sue lettere Pavelić, ad esempio, invitò gli ustascia residenti in Croazia a partecipare alle elezioni del parlamento del 1935 e a votare il leader del Partito dei Contadini Croato Vladko Maček (Kvaternik, 1995, p. 220).Višnja Pavelić racconta persino che sua madre ha incontrato degli agenti croati dentro e fuori I'Italia per scambiare delle informazioni sulla situazione politica in lugoslavia e per parlare delle mosse politiche e strategiche da intraprendere:

"Quando papà era in carcere, toccava a lei. Alcune volte, diciamo, (loro) sono venuti a Roma, da pellegrini, e il papà era in carcere. E poi la mamma aveva le notizie, allora, andava, ad esempio, a Roma, e ci parlava con delle persone, perché esse erano venute a Roma proprio per sentire cosa succedeva in Italia, perché in quei tempi non c'era nessun contatto. Papà era in carcere. Ogni contatto con la Croazia era interrotto. Poi sono venuti e si sono incontrati con mia madre (...) Naturalmente anche il documento di viaggio portava un nome falso, quando lei è venuta in Svizzera per vedermi e altro, e ci ha fatto un rapporto. E tutto questo era in un tempo in cui il papà era in carcere."

Anche la polizia italiana, che la spiava continuamente, si rese conto del fatto che Marija Pavelić era una donna di altissima intelligenza e astuzia. ${ }^{6}$ I rapporti di sorveglianza confermano l'impegno politico di Marija Pavelić. Avrebbe compiuto delle missioni delicate per il movimento e si sarebbe avvalsa di ogni mezzo per sostenere suo marito nei suoi affari politici, come si dice nel seguente verbale del dicembre 1937:

"La signora Pavelić è assai astuta e ha sempre cercato di fiancheggiare il marito nella sua attività politica, compiendo missioni talvolta assai delicate, specialmente per mantenere $i$ contatti epistolari con fuorusciti croati residenti all'estero. Essendo assolutamente certo che il Pavelić ha in questi ultimi tempi cercato di riprendere la sua attività politica con l'estero, ed essendo sfuggite al controllo lettere che sicuramente gli sono pervenute, si ha motivo che la Pavelić suddetta valendosi di amicizie contratte durante la sua permanenza costà, abbia indotto qualche persona a prestarsi a riceverle la corrispondenza per poi consegnarla." 7

Marija Pavelić s'impegnava costantemente per sostenere la presa di potere di suo marito, rafforzando la sua posizione tra le file del movimento ustascia e propagandando la sua legittimazione per la funzione di capo, ossia rendendolo una figura di culto di 'duce' politico carismatico come quella di Hitler nella Germania nazionalsocialista e di Mussolini nell'Italia fascista. A quanto dichiarava il generale Ivan Perčević, Marija Pavelić elogiava suo marito eccessivamente davanti ai suoi compagni, affermando che loro avrebbero ancora visto la genialità e la forza del politico Pavelić. ${ }^{8}$

Le conseguenze del rafforzamento dell'autorità e del potere di Pavelić si facevano sentire per quelli ustascia che erano caduti in disgrazia agli occhi dei coniugi per essersi esposti troppo sulla scena politica. Slavko Kvaternik era sicuramente visto negativamente dai Pavelić, avendo letto la proclamazione dello Stato Indipendente di Croazia alla radio zagabrese il 10 aprile 1941 al posto del capo ustascia, mentre quest'ultimo si trovava ancora in Italia e fu colto dal messaggio visibilmente impreparato. Sebbene Kvaternik l'avesse fatto a nome di Pavelić, la coppia ne sarebbe rimasta molto amareggiata. Nella loro casa, la gioia per la proclamazione dello Stato quel giorno non sarebbe stata una gioia sentita (Kvaternik, 1995, p. 31ss).Ante Moškov riferì che Marija Pavelić aveva attaccato Kvaternik per l'avvenimento alcuni anni dopo, chiedendogli se il generale d'armata non avrebbe potuto aspettare con la proclamazione dello Stato

\footnotetext{
5"Dok je tata bio u zatvoru, onda je ona morala nastupiti. Koji puta recimo, dolazili su u Rim, kao hodočasnici, onda, a otac je bio u zatvoru. I onda je mama poruke_, ovaj, išla je u Rim, na primjer i tamo je razgovarala sa ljudima, jer su namjerno dolazili da čuju šta se događalo u Italiji, jer nije bilo onda veze. Tata je bio u zatvoru. Bila je podpuno prekinuta svaka veza sa Hrvatskom. Onda su dolazili $i$ moja mama se sastajala s njima. (...) Naravno putovnica joj je bila na krivo ime, kad je dolazila u Švicarsku mene posjetit i tako, i tamo obavljala koju, koju poruku. A to je sve bilo u vrijeme, dok je tata bio u zatvoru." Intervista con Višnja Pavelić il 23. 2. 2008.

${ }^{6}$ Archivio Centrale dello Stato (ACS), Pubblica Sicurezza (PS), Ercole Conti, b. 16, 30. 1. 1938.

7 Ibid., 23.12. 1937.

${ }^{8}$ HDA, SDS RSUP SRH -1561, Ustaški pokret i NDH, busta 23, sig. 013.0.4, pratica Ante Pavelić, 16. 3. 1947.
} 
Indipendente di Croazia, finché il 'poglavnik' non fosse tornato a Zagabria. ${ }^{9}$ Kvaternik, che era capo dell'esercito croato dal 1941, sentiva le conseguenze di quest'avversione dei Pavelić, quando nel 1942 fu destituito e dovette fuggire in Slovacchia per una lite con Pavelić.

\section{La donna leader dello Stato Indipendente di Croazia (1941-1945)}

L'immagine pubblica della consorte del 'poglavnik' nello Stato Indipendente di Croazia era quella di una madre, casalinga e moglie premurosa. La stampa faceva vedere delle fotografie di lei in compagnia di suo marito a teatro ${ }^{10}$, in occasione di eventi pubblici e di feste popolari ${ }^{11}$, con la sua famiglia ${ }^{12} \mathrm{e}$ anche con un gruppo di orfani dell'Istituto di Formazione degli Ustascia per Ragazzi i3 . II fatto che lei non era solo l'ombra di suo marito, ma che s'immischiava bensì negli affari politici, spunta solo in un'immagine in La Nuova Croazia (Nova Hrvatska) nella quale si vede come lei conversa con tre operai seduti in cerchio. II giornale vi aggiunge la seguente didascalia:

"La moglie del Poglavnik, conversando con alcuni operai croati che hanno partecipato alla cena tenuta dal Poglavnik in onore degli operai per la Festa del Lavoro. La moglie del Poglavnik sta seguendo le discussioni degli operai su questioni sociali con particolare interesse e comprensione." 14

Una dimostrazione così palese della vicinanza della moglie del 'duce' al popolo era sicuramente inappropriata in quanto avrebbe potuto pregiudicare il ruolo simbolico di Pavelić. Tuttavia né lui né lei avevano un'intenzione del genere.

Come quasi tutte le mogli di capi di Stato, anche lei e le sue figlie Višnja e Mirjana s'impegnavano nelle opere di carità. Era patrocinatrice e fondatrice dell'Istituto di Formazione Ustascia per Ragazzi, costituito nell'aprile 1942, un istituto elitario privato che si trovava vicino alla villa zagabrese dei Pavelić. Una cinquantina di orfani croati in età da scuola elementare, educati dalle suore di San Vincenzo de' Paoli, erano destinati a svolgere servizio come funzionari ustascia o come militari una volta compiuta la loro formazione. A tale scopo, il programma scolastico comprendeva, oltre alle lezioni regolari, anche una formazione militare. La stampa chiamava i bambini "il piccolo battaglione del Poglavnik" (mala Poglavnikova bojna). ${ }^{15}$ La vicinanza geografica e personale a Pavelić e alla sua famiglia era una possibile garanzia per la formazione di seguaci fedeli allo Stato e al 'duce'. L'idea di assicurarsi la simpatia degli orfani era efficace, da un punto di vista psicologico, perché era facile suscitare in loro la nostalgia delle figure paterna e materna. Alcuni ex-ragazzi cadetti continuarono a sentire il legame anche decenni dopo la fine della guerra (Kovačević, 2004). La stampa loro contemporanea riportava dell'amore con il quale la "madre premurosa" Marija Pavelić si occupava dei bambini. ${ }^{16} \mathrm{Da}$ sarta esperta, creava le divise blu e andava spesso a far loro visita (Kovačević, 2004, p. 96).

Secondo Slavko Kvaternik però, la Pavelić aveva solo fatto finta di adottare quel ruolo femminile stereotipato, perché lo riteneva adatto a una società patriarcale. ${ }^{17}$ In realtà, lei giocava un ruolo molto dominante ed esercitava un controllo sugli affari politici di Pavelić: dal momento della costituzione dello Stato Indipendente Croato, i conoscenti più stretti dell'élite ustascia chiamavano Marija Pavelić "la duce" (Poglavnikovica). Quel termine era sicuramente opportuno, vista la forza del suo impatto sia nella politica che sui media, nell'arte e persino nell'edilizia. A quanto diceva Ante Moškov, lei aveva esercitato il suo potere soprattutto nella provincia di Prigorje, con sede amministrativa a Zagabria. In fondo, il generale e capo di provincia Viktor Prebeg rispondeva a lei e aveva il compito di esaudire i suoi vari desideri. Pur essendo stato favorito con tutta la sua famiglia per un periodo molto lungo, cadde in disgrazia - come succedeva a tanti funzionari - apparentemente solo perché si era fatto fare delle borse con un disegno uguale a quello della Pavelić. Una faccenda che le stava molto a cuore era la modernizzazione della provincia di Prigorje e del suo capoluogo Zagabria. Per motivi estetici, avrebbe voluto limitare l'ulteriore promozione del consumo d'alcol attraverso la pubblicità e,

9 "Zar nije vojskovodja mogao pričekati sa proglašenjem Nezavisne Države Hrvatske dok ne dodje poglavnik u Zagreb?"HDA, SDS RSUP SRH -1561, Ustaški pokret i NDH, kut. 37, sig. 013.0.58, pratica di Ante Moškov, senza data.

10 Nova Hrvatska, 10. 4. 1943/86.

11 Ustaškinja, 10. 10. 1942/7, p. 2.

12 Hrvatski narod, 20. 4. 1941/67.

13 Ustaškinja, 10. 4. 1943/1, p. 18-19.

14"Poglavnikova gospodja zadržala se u razgovoru s hrvatskim radnicima, koji su prisustvovali večeri što ju je Poglavnik priredio u čast radnika, uoči blagdana rada. Poglavnikova gospodja s osobitom pažnjom i razumijevanjem prati izlaganja radnika o njihovim društvovnim pitanjima." Nova Hrvatska, 5. 5. 1942/104.

15 Ustaškinja, 10. 4. 1943/1, p. 18.

16 Ustaškinja, 10. 4. 1943/1, p. 18-19.

17 HDA, SDS RSUP SRH - 1561, Ustaški pokret, busta 24, sig. 013.0.4, pratica Ante Pavelić, senza data. 
quindi, ordinò di rimuovere alcuni cartelloni pubblicitari da Zagabria. Questi furono sostituiti con cartelloni enormi sui quali si vedeva suo marito seduto su un cavallo. Le sue modifiche urbanistiche non si limitavano ad azioni come queste però: s'interessava anche dei lavori di risanamento e d'edilizia nella provincia di Prigorje, determinando quali edifici si sarebbero ricostruiti e provvedendo alla loro demolizione una volta che aveva dato il suo consenso. Tutto si sarebbe dovuto orientare a suo gusto. Ciò si fece sentire anche sugli impiegati di una stazione radio che, a mezzanotte - sotto minaccia d'arresto - sarebbero stati costretti a cambiare musica.La sua maggiore intenzione era di tenere suo marito al potere con tutti i mezzi disponibili e di non esporlo a nessun rischio. Per controllare che il cibo non contenesse del veleno, sorvegliava pure la preparazione dei pasti. Nella famiglia dei Pavelić si prestava grande attenzione a far servire il capo dello Stato solo da sua moglie o da sua figlia Višnja. Assunsero una cuoca italiana che era venuta con loro dall'Italia, per non doversi fidare di una croata. Anche questa impiegata, però, era controllata durante le sue attività da Marija Pavelić. ${ }^{18}$

Per di più, s'impegnò a creare l'immagine di culto di suo marito: II fotografo Mario Medeott, ad esempio, descrive Pavelić come un uomo dall'aria criminosa sui ritratti. Queste immagini, però, non piacevano a Marija Pavelić, per cui chiese al fotografo di ripetere le sessioni, finché non le venne l'idea di far rallegrare suo marito con delle barzellette, mentre posava, per rendere le foto più piacevoli (Vojinović, 1988, p. 16).

Tutte le rappresentazioni che cercano di mostrare il carattere della moglie del dittatore lasciano aperta una domanda chiave: fino a che punto Marija Pavelić influiva sulla politica di suo marito e fino a che punto lui si faceva influenzare da sua moglie nelle decisioni politiche? Esercitò il suo potere da cosiddetta Poglavnikovica nella nomina o nel licenziamento di funzionari e ministri. Se qualcuno fosse caduto in disgrazia, ne sarebbe stato discusso in famiglia, con il marito 0 anche con la figlia maggiore Višnja. A volte, insultava le persone da allontanare davanti ai rispettivi confidenti. Grazie alla Sezione Femminile del Movimento Ustascia (Ženska loza hrvatskog ustaškog pokreta), possedeva anche una rete di spie e denuncianti di cui rimasero vittime alcuni cittadini zagabresi. La comandante dell'organizzazione femminile, Irena Javor, era la confidente alla quale competeva questo compito. ${ }^{19}$

Da moglie di un criminale di guerra, Marija Pavelić non sfuggì al rimprovero, che fu pronunciato, tra l'altro, da Slavko Kvaternik, Ante Moškov, Ivan Perčević e Vladimir Košak, di aver sostenuto azioni criminali nello Stato Indipendente di Croazia. Slavko Kvaternik la definì persino come una forza oscura che aveva le stesse colpe di suo marito per i crimini commessi, in particolare riguardo agli omicidi commessi tra la popolazione serba. ${ }^{20}$ Rifiutò anche il suo aiuto a un'amica di gioventù il cui marito si trovava in pericolo di morte (Lasić, 2000, p. 178).La sua origine ebraica da parte materna non le impedi di esprimere il parere secondo cui gli ebrei meritavano di essere deportati nei lager. ${ }^{21}$ Secondo le Leggi Razziali, introdotte nello Stato ustascia nel 1941, anche Marija Pavelić sarebbe stata classificata come "mezzebrea" (Halbjüdin), indipendentemente dal fatto che sua madre si fosse assimilata o no. Višnja Pavelić afferma che sua nonna era ebrea, sostenendo, però, che si fosse fatta battezzare, come anche sua madre. ${ }^{22}$ II fatto che Marija Pavelić fosse di origine ebraica era noto anche ai nazionalsocialisti: Nei ricordi del generale Edmund Glaise von Horstenau, è chiamata cinicamente "mezzebrea" o "Kalle", che in yiddish significa sposa o moglie (Broucek, 1988, p. 506 e p. 511).Una donna di "mezzosangue ebraico" (jüdischer Mischling)al fianco del 'poglavnik' era tollerata dai tedeschi solo per motivi politici.

Slavko Kvaternik continua a riportare che Marija Pavelić aveva un carattere più forte di quello di suo marito. Quando lui si arrese, lei cercò di tirarlo su di morale, riempiendolo di nuova energia. L'ex-generale d'armata descrisse il dominio nello Stato Indipendente di Croazia addirittura come un "despotismo familiare" (obiteljski despotizam), per l'influsso che la moglie esercitava sulla personalità e sulle azioni politiche di Ante Pavelić. Per quanto riguarda la domanda sulla misura nella quale Marija Pavelić ha influito sulla politica del capo degli ustascia, Moškov fa un passo ancora più in avanti, sostenendo che lei non lo avrebbe influenzato, non era necessario, perché loro si completavano come due persone in una:

"No, Mara [=Marija Pavelić] non ha esercitato nessun influsso su Pavelić, tra di loro c'era una comprensione assoluta nelle domande più sostanziali; avevano gli stessi desideri e le stesse intenzioni; i loro pensieri e i

\footnotetext{
18 Ibid., 14.5.1947.

19/bid.

20 HDA, SDS RSUP SRH-1561, Ustaški pokret, busta 24, sig. 013.0.4, pratica Ante Pavelić, deposizione di Slavko Kvaternik, senza data.

${ }^{21}$ lbid., deposizione di Ante Moškov, senza data.

22 Intervista con Višnja Pavelić a Madriddel 23.2.2008.
} 
loro piani erano indubbiamente identici, i loro caratteri si completavano, il loro metodo, il modo di lavorare erano pensati insieme."23

Al pubblico recitavano, continua, il loro ruolo stereotipato da marito e moglie con successo, ma dietro le quinte facevano politica insieme.

\section{Conclusione}

La tre volte madre Marija Pavelić era assai influente nell'élite politica degli ustascia e decideva - con l'obiettivo di rinforzare la posizione di suo marito - chi sarebbe rimasto sulla scena politica, chi sarebbe stato spostato e persino chi sarebbe stato destituito. Influiva sui media, faceva pedinare la cittadinanza zagabrese e faceva denunciare i cittadini caduti in disgrazia, badava all'aspetto della capitale, perché lo riteneva balcanizzato ecc. Nonostante la sua alta intelligenza, la sua sfera d'influenza e il suo potenziale da personalità leader, recitava al pubblico la parte della moglie disinteressata negli affari politici al fianco di Ante Pavelić per corrispondere all'ideale femminile degli ustascia.

\section{Bibliografia}

Intervista

Intervista con Višnja Pavelić a Madrid del 23./24. 2. 2008.

Archivi

Archivio Centrale dello Stato (ACS), Pubblica Sicurezza (PS), Ercole Conti.

Hrvatski Državni Arhiv (HDA), Služba državne sigurnosti republičkog sekretarijata za unutrašnje poslove Socijalističke Republike Hrvatske (SDS RSUP SRH) - 1561, Ustaški pokret, busta 24, sig. 013.0.4, pratica di Ante Pavelić.

Hrvatski Državni Arhiv (HDA), Služba državne sigurnosti republičkog sekretarijata za unutrašnje poslove Socijalističke Republike Hrvatske (SDS RSUP SRH) - 1561, Ustaški pokret i NDH, busta 37, sig. 013.0.58, pratica di Ante Moškov.

Periodica

Hrvatski narod.

Nova Hrvatska.

Ustaškinja.

Letteratura

Becherelli, A. (2012). Italia e Stato Indipendente Croato (1941-1943), Roma: Nuova cultura.

Broucek, P. (a cura di) (1988). Ein General im Zwielicht. Die Erinnerungen, Edmund Glaises von Horstenau, Deutscher Bevollmächtigter General in Kroatien und Zeuge des Untergangs des „Tausendjährigen Reiches“, libro 3, Vienna: Böhlau Verlag.

Jareb, J. (1982). Političke uspomene i rad dra Branimira Jelića, Cleveland: M. Šamija.

Kovačević, B. (2004). Plavi dječaci, Zagabria: Branimir Kovačević.

Kvaternik, E. D. (1995). Sjećanja i zapažanja 1925.- 1945. Prilozi za hrvatsku povijest, a cura di Jere Jareb, Zagabria: Naklada Starčević.

Lasić, S. (2000). Autobiografski zapisi, Zagabria: Nakladni zavod Globus.

Pavelić, V. (2001). Martin Lovrenčević, Grič, 1, 25-32.

Tomasevich, J. (2010). Rat i revolucija u Jugoslaviji 1941.-1945. Okupacija i kolaboracija, Zagabria: EPH Media.

Vojinović, A. (1988). Ante Pavelić, Zagabria: Centar za informacije i publicitet.

23 "Ne, Mara [=Marija Pavelić] nije utjecala na Pavelića; izmedju njih je postojalo potpuno razumijevanje u osnovnim pitanjima; oni su imali iste želje i namjere; njihove misli i planovi su bez sumnje zajednički, njihovi su se karakteri međusobno popunjavali, njihov metod, načinrada bio je zajednički smišljen." HDA, SDS RSUP SRH-1561, Ustaški pokret, busta 24, sig. 013.0.4, pratica Ante Pavelić, deposizione di Ante Moškov, senza data. 